\title{
Hippocampus and amygdale MRI studies: anatomy, current concepts of physiology and methological limitations Asterios Fotiadis*2, Zoe Nassika ${ }^{3}$, Jannis Nimatoudis ${ }^{1}$, Athanasios Karavatos ${ }^{2}$ and George Kaprinis ${ }^{1}$
}

\author{
Address: ${ }^{1} \mathrm{C}^{\prime}$ Department of Psychiatry, Aristotle University of Thessaloniki, Thessaloniki, Greece, ${ }^{2} \mathrm{~A}^{\prime}$ Department of Psychiatry, Aristotle University \\ of Thessaloniki, Thessaloniki, Greece and ${ }^{3}$ Department of Psychiatry, University of Thessaly, Larissa, Greece \\ * Corresponding author
}

\author{
from International Society on Brain and Behaviour: 3rd International Congress on Brain and Behaviour \\ Thessaloniki, Greece. 28 November - 2 December 2007 \\ Published: 17 April 2008 \\ Annals of General Psychiatry 2008, 7(SuppI I):S342 doi:I0.I I86/I744-859X-7-SI-S342
}

This abstract is available from: http://www.annals-general-psychiatry.com/content/7/SI/S342

(c) 2008 Fotiadis et al.; licensee BioMed Central Ltd.

\section{Background}

Recent advances in MRI technique made possible the study of small cerebral structures, such as the hippocampus and amygdala. The aim of the study is to present the current MRI finding (anatomical boundaries, physiology and methodological problems) of the hippocampus and amygdala.

\section{Materials and methods}

Selective review of MRI and post- mortem studies between 2000-2006 in Medline. Preferential focus on review articles and studies including large number of participants.

\section{Results}

We present figures and MRI images to define the landmarks for hippocampus and amygdala, especially the relation between the temporal horn of the lateral ventricles. The current concept of amygdala physiology is that, with it's connections to medial prefrontal cortex, has a central role in emotional information processing (for example recognition of fearful stimuli) and also regulates autonomic responses. The hippocampus is the center of memory and learning (especially verbal) and it also involved in emotional processing of stimuli in working memory. The methodological problems rise from their small volumes, the total (right and left hemisphere) volume of each structure being $<0,5 \%$ of whole brain volume. Therefore many studies measure the hippocampal plus amygdala complex, which can be divided to posterior (mainly hippoc- ampal) and anterior portion (mainly amygdala). Their small volume also results in reduced interrater reliability.

\section{Conclusions}

Both hippocampus and amygdala sustain functions that pertain to complex emotional processing. Their small size still results in difficulties of delineation, especially from the overlapping temporal horn of the lateral ventricles, in post-mortem studies. 